\title{
The Immunoreactivity of PI3K/AKT Pathway After Prenatal Hypoxic Damage
}

\author{
HUISUN WANG ${ }^{1}$, YOONYOUNG CHUNG ${ }^{2}$, SUN-KYOUNG YU ${ }^{3}$ and YONGHYUN JUN ${ }^{2}$ \\ ${ }^{1}$ Department of Neurosurgery, Chosun University Hospital, Gwang-ju, Republic of Korea; \\ ${ }^{2}$ Department of Anatomy, School of Medicine, Chosun University, Gwang-ju, Republic of Korea; \\ ${ }^{3}$ Department of Oral Anatomy, School of Dentistry, Chosun University, Gwang-ju, Republic of Korea
}

\begin{abstract}
Background/Aim: There is no consensus on the effect of hypoxia on neurogenesis. In this study, we investigated the immunoreactivity of BDNF and PI3K/Akt signaling after uterine artery ligation in pregnant rats. Materials and Methods: Unilateral uterine artery ligation was performed at 16 days of gestation ( $\mathrm{dg}$ ). Fetuses from one horn with ligated artery were allocated to the hypoxic group. Immunohistochemistry was performed with primary antibodies; NeuN, BDNF, PI3K, Akt and phospho-Akt (pAkt). Results: The densities of NeuN-and BDNF-immunoreactive (IR) cells in the cerebral cortex were lower in the hypoxic fetuses than in the controls at $21 \mathrm{dg}$. The density of PI3K and pAkt-IR cells in the cortex of the hypoxic group significantly decreased. The results in dentate gyrus were similar to the results in the cerebral cortex. Conclusion: Prenatal hypoxia reduced Akt phosphorylation, which affected neuronal survival in the cortex and dentate gyrus.
\end{abstract}

Prenatal hypoxia causes abnormal brain development. The brain volumes are reduced in low birth weight infants due to hypoxic conditions (1). White matter and myelin sheath are vulnerable to hypoxic conditions $(2,3)$. This abnormal fetal brain development is associated with the imbalance between the increasing oxygen requirement and substrate in a developing fetus (4). Prenatal hypoxia is a symptom of chronic placental insufficiency (CPI). CPI leads to neurological disorders including cerebral palsy (5), cognitive deficit (6), learning/memory problems (7), and schizophrenia (8).

This article is freely accessible online.

Correspondence to: Yonghyun Jun, Department of anatomy, School of medicine, Chosun University, 375 Seosuk-dong, Dong-Gu, Gwangju 501-759, Republic of Korea. Tel: +82 1028862973, Fax: +82 0622341474, e-mail: jyh1483@chosun.ac.kr

Key Words: Prenatal hypoxia, neurogenesis, Akt, pAkt, PI3K.
Prenatal hypoxia affects neurogenesis, which is a process of generation of new neurons from progenitor neuronal stem cells. There is no consensus on the effect of hypoxia on neurogenesis. It was reported that prenatal hypoxia induced neurogenesis in developing rat brain (9). In addition, progenitor cells in the subventricular zone (SVZ) were vulnerable to ischemic insult in the fetal mouse (10). SVZ is a neurogenic zone in the cerebrum, which also includes the olfactory bulb and the subgranular layer of the hippocampus (SGZ).

In our previous study, we showed that cell proliferation in SVZ and dentate gyrus (DG) was not different between normal and hypoxic fetuses, but cell survival in the cerebral cortex and DG of the hypoxic group was different from the normal group. In addition, the number of brain-derived neurotrophic factor (BDNF)-immunoreactive (IR) cells was decreased in the cortex and DG (11).

BDNF is important in neuronal cell proliferation, growth and survival (12). BDNF activates phosphoinositide 3-kinase $(\mathrm{PI} 3 \mathrm{~K}) /$ protein kinase B (Akt) signaling (13). In this study, we investigated the immunoreactivity of PI3K and Akt phosphorylation (pAkt) after hypoxic damage.

\section{Materials and Methods}

Animal surgery. All animal experiments were approved by the Chosun University Institutional Animal Care and Use Committee (approval number CIACUC2015-A0018). Sprague-Dawley (SD) rats were obtained from a certified breeder (Damul Laboratory Animals, Republic of Korea). Rats were mated and checked for the vaginal plug to confirm the pregnant status. Unilateral uterine artery ligation was designed as reported in a previous study (14). Briefly, animals were anesthetized with Zoletil $(10 \mathrm{mg} / \mathrm{kg}$; Virbac, France) and xylazine (0.15 mg/kg; Bayer, Germany), via intramuscular injection at 16 days of gestation (dg). After shaving the abdominal region below the umbilicus, a midline incision was performed under aseptic conditions. The fat pad in the uterine horn was revealed, and the uterine artery was tied with silk sutures (4/0). After the procedure, the surgical site was sterilized with a povidone-iodine solution (Green Medical Co., Ltd., Tokyo, Japan). 

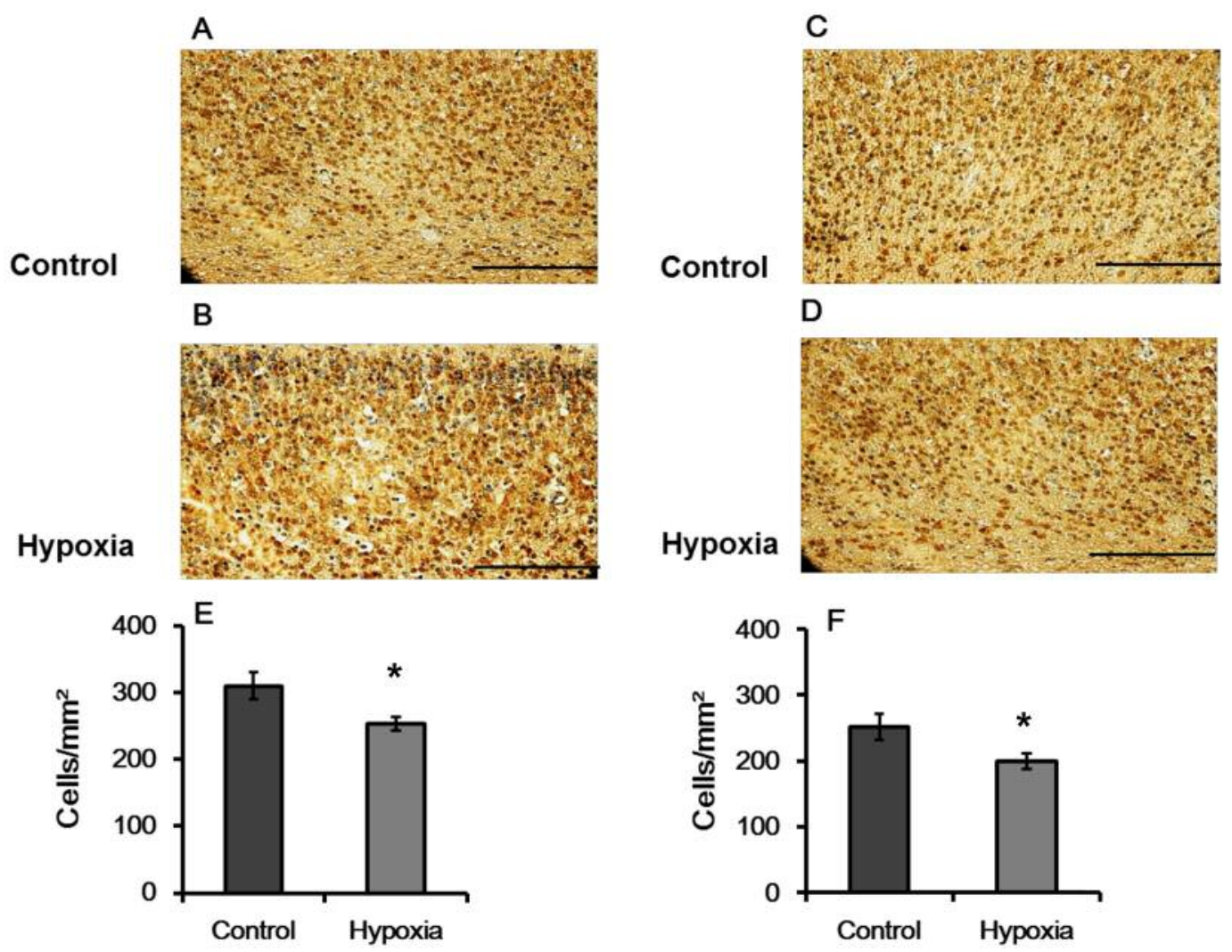

Figure 1. Representative photomicrographs of the immunoreactivity for neuronal nuclei (NeuN; A and B) and brain-derived neurotrophic factor $(B D N F ; C$ and D) in the parietal cortex of rats at 21 days gestation $(\mathrm{dg})$. Scale bars $=100 \mu \mathrm{m}$. The densities of the NeuN-immunoreactive (IR; E) and BDNF-IR $(F)$ cells in the cerebral cortex were significantly decreased in the hypoxic fetuses compared with the controls. The data are expressed as mean \pm standard error of the mean (SEM). ${ }^{*} p<0.05$

Tissue preparation. The rat fetuses were delivered by cesarean sections at $21 \mathrm{dg}$ from pregnant rats $(\mathrm{n}=14)$ that were subjected to hypoxic injuries. Fetuses from one horn with the unligated uterine artery were allocated to the control group $(n=47)$ and those from the other horn with the ligated artery were allocated to the hypoxic group $(\mathrm{n}=45)$. After removing fetuses from the uterine horn, brains were stored in $4 \%$ paraformaldehyde (PFA) solution. Fetal cerebrums were separated from brain stems and fixed in 4\% PFA at $4^{\circ} \mathrm{C}$. After two days, the cerebrum was cleaned with distilled water and dehydrated through a graded ethanol series and then the cerebrums were embedded in paraffin. Serial coronal sections of 12 $\mu \mathrm{m}$ thickness were cut and the sections were chosen at regular intervals from each animal. These sections were placed on gelatincoated slides (Fisher Scientific, PA, USA).

Immunohistochemistry. The sections were deparaffinized and washed in $0.1 \mathrm{M}$ phosphate buffered saline (PBS; pH 7.4). Antigen retrieval was performed by heating the sections in a microwave with $0.01 \mathrm{M}$ sodium citrate buffer ( $\mathrm{pH}$ 6.0). After cooling, the sections were put in $0.3 \%$ hydrogen peroxide solution for 20 min to block endogenous peroxidase. The sections were then incubated with one of the following primary antibodies overnight at $4{ }^{\circ} \mathrm{C}$ : mouse antineuronal nuclei (NeuN; 1:100; Millipore, Massachusetts, USA), rabbit anti-BDNF (1:50; Santa Cruz Biotechnology, TX, USA), rabbit anti-Akt (1:50; Santa Cruz, TX, USA), rabbit anti-phosphoAkt (1:50; Santa Cruz, TX, USA). The next day, the sections were washed with PBS and treated with appropriate secondary antibodies, depending on the type of primary antibody. Immunoreactivity was visualized using avidin-biotin-peroxidase $(\mathrm{ABC})$ detection system (Vectastain ABC Elite Kit, Vector Laboratories, Burlingame, CA, USA). Thionin counterstaining was performed, and the sections were coverslipped using PolyMount mounting medium (Polysciences, Warrington, PA, USA).

Quantification of IR cells. The slides were observed using a light microscope (Olympus BX41, USA) connected to a digital CCD camera. The Image-Pro Plus 7.0 image analysis software program (Media Cybernetics, Inc., Rockville, MD, USA) was used by three investigators to measure the densities of the NeuN-, BDNF-, PI3K-, Akt-, and phospho-Akt (pAkt)-IR cells (cells $/ \mathrm{mm}^{2}$ ). The density of positive cells was counted within five randomly chosen defined field similarly as in a previous study (15).

Statistical analysis. All data were measured using Statistical Package for Social Sciences (Information Analysis Systems, SPSS, USA). All measurements were analyzed between the control and hypoxic groups using Student's $t$-test. The level of statistical significance was set at $p<0.05$. 

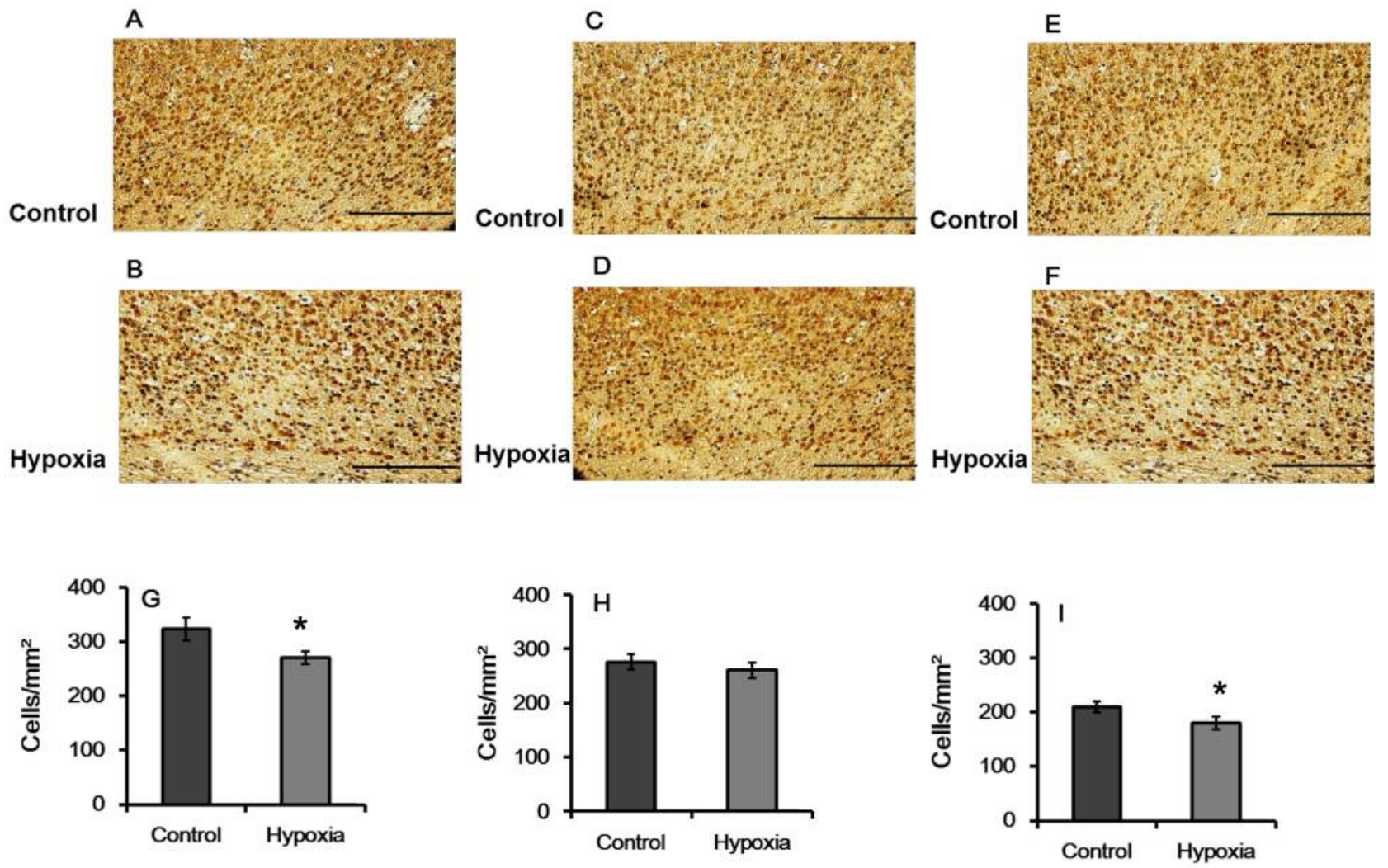

Figure 2. Representative photomicrographs of the immunoreactivity for phosphoinositide 3-kinase (PI3K; A and B), protein kinase B (Akt; $C$ and $D$ ), and phospho-Akt (pAkt; $E$ and $F$ ) in the parietal cortex of rats at $21 \mathrm{dg}$. Scale bars $=100 \mu \mathrm{m}$. The densities of the PI3K-IR $(G)$, Akt-IR $(H)$, and pAkt-IR (I) cells in the cerebral cortex of the control and hypoxic fetuses are shown, with significant decreases in the densities of the PI3K-IR and pAKT-IR cells in the hypoxic fetuses compared with the controls. The data are expressed as mean $\pm S E M . * p<0.05$.

\section{Results}

Cerebral cortex. The densities of NeuN- and BDNF-IR cells in the cerebral cortex were lower in the hypoxic fetuses $(\mathrm{n}=10)$ than in the controls $(\mathrm{n}=10)$ at $21 \mathrm{dg}(p<0.05$; Figure $1)$. The density of PI3K-IR cells in the cortex significantly was reduced in hypoxic fetuses (Figure 2). Similarly, the density of pAkt-IR cells in the hypoxic group was lower than the density of that in the control group (Figure 2). However, there was no difference between normal and hypoxic fetuses in the density of Akt-IR cells in the cortex (Figure 2).

Dentate gyrus. The results in dentate gyrus were similar to the results in the cerebral cortex. The densities of NeuN and BDNF-IR cells were decreased in the hypoxic group $(n=10)$ compared with those in the control group $(n=10)$ (Figure 3$)$. The densities of PI3K and pAkt-IR cells were lower in the hypoxic group than in the control group (Figure 4). Interestingly, the density of Akt-IR cells was not different between the control and hypoxic groups (Figure 4).

\section{Discussion}

Hypoxic condition was made by unilateral uterine artery ligation of pregnant rats. This condition significantly decreased uterine blood flow and fetal body weight (16). In our previous study, we examined two regions, cerebral cortex, and dentate gyrus of the hippocampus and determined the neuronal loss and decrease in neurotrophic factor level (11). Therefore, in this study, we investigated the immunoreactivity of NeuN, BDNF, PI3K, Akt, and pAkt in the same two regions mentioned above. The densities of NeuN-, BDNF-, PI3K- and pAkt-IR in the cerebral cortex and dentate gyrus of hypoxic group fetuses were lower than in that of the control group.

BDNF is an important neuronal factor that protects from ischemic brain injury. It is a member of the neurotrophin (NT) gene family, which includes nerve growth factor, neurotrophin-3 (NT-3) and NT-4. NT genes are involved in modulating the survival and development of neurons (17). BDNF synthesis is regulated by calcium influx (18). Under 

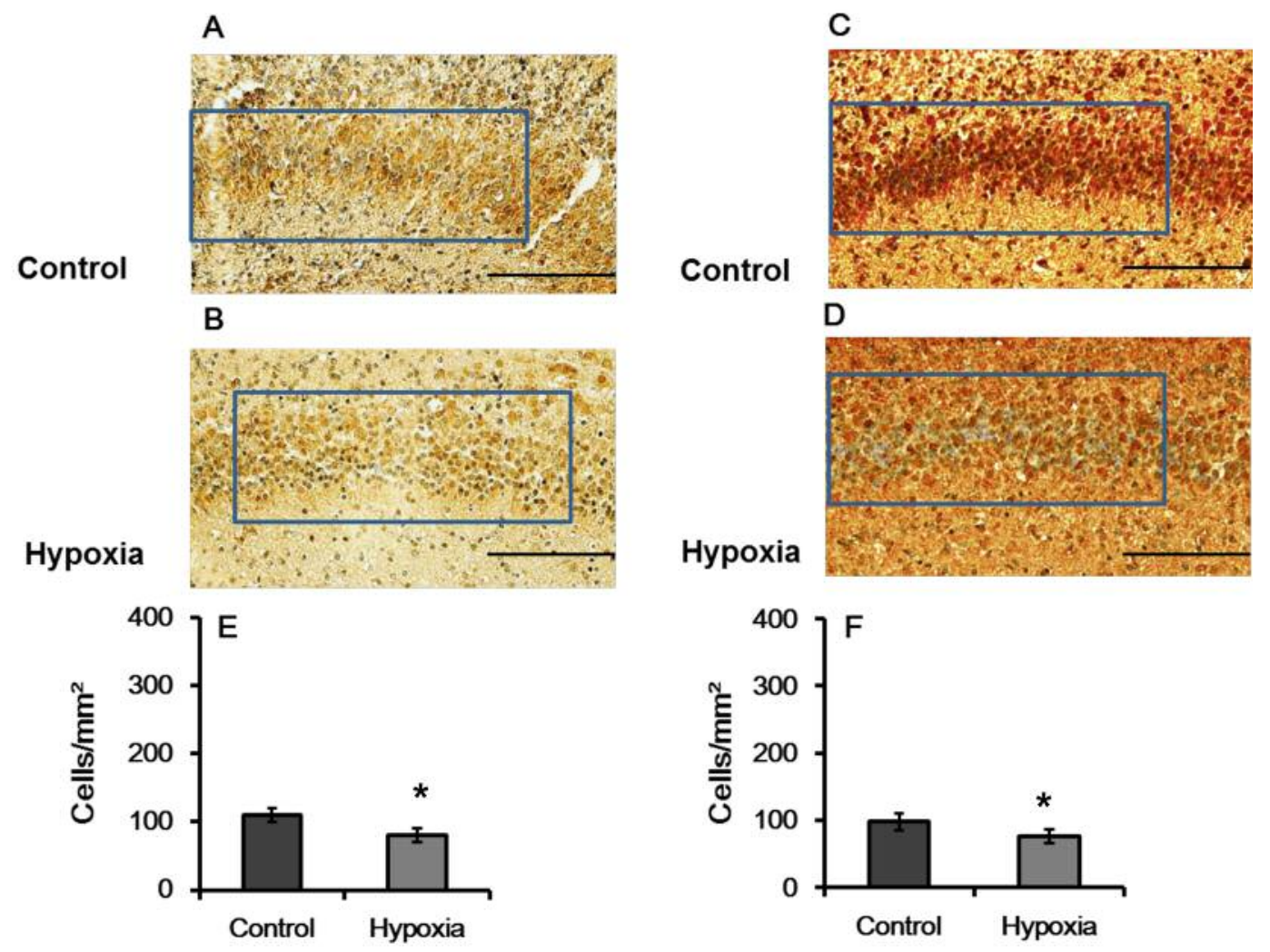

Figure 3. Representative photomicrographs of the immunoreactivity for NeuN (A and B) and BDNF (C and D) in the dentate gyrus (outlined by blue boxes) of rats at $21 \mathrm{dg}$. Scale bars $=100 \mu \mathrm{m}$. The densities of the NeuN-IR $(E)$ and BDNF-IR $(F)$ cells in the dentate gyrus were significantly decreased in the hypoxic fetuses compared with the controls. The data are expressed as mean \pm SEM. ${ }^{*} p<0.05$.

hypoxic condition, calcium homeostasis is not maintained (19), suggesting that prenatal hypoxia reduces the density of BDNF-IR cells. The survival function of BDNF is regulated by activation of two cell surface receptors, TrkB and p75 neurotrophin receptor (20). BDNF activates several intracellular signaling pathways via TrkB receptor, for example, PI3K/Akt pathway, which affects the function of the developing nervous system (13).

Some recent studies suggest that PI3-kinase is an intracellular transducer of survival signals and is initiated by diverse growth factors (21-23). Akt, known as protein kinase $\mathrm{B}$, is a protein kinase associated with survival signals that are modulated by downstream kinase of PI3-K in growth factorinduced signaling cascades (24). Akt is activated by PI3kinases and binds phosphorylated lipids membrane (25). $\mathrm{PI} 3 \mathrm{~K} /$ Akt signaling is involved in cell proliferation, cell migration, survival, and dendritic growth through mTOR (26). This signaling was activated to varying degrees of phosphorylation of BDNF and activation of AKT, which promoted neuronal survival and prevented apoptosis through under cellular mechanism such as phosphorylation of Bad (27).
In neonatal rats, phospho-Akt is decreased during hypoxia but the total Akt level is unchanged, similarly to our results (28). Hristelina et al. showed that p-Akt signal was lower immediately after recovery from $2 \mathrm{~h}$ of hypoxia, compared to the normoxic group (29). These reports suggest that energy for activation of Akt to compensate for oxidative stress was not enough under hypoxic conditions. Another report showed a temporary increase of Akt phosphorylation (30). However, the mechanism of Akt phosphorylation during or after hypoxia is not clear. Ouyang suggested that cytochrome $\mathrm{c}$ is released from the mitochondria after Akt activation in a global ischemia model (31). Some studies reported regional differences in phosphorylation of Akt after hypoxic damage $(24,25)$, which might be associated with the severity of ischemic insults (25).

\section{Conclusion}

Prenatal hypoxic damage reduced neuronal cell survival in cerebral cortex and dentate gyrus. This phenomenon was caused by decreasing Akt phosphorylation. 


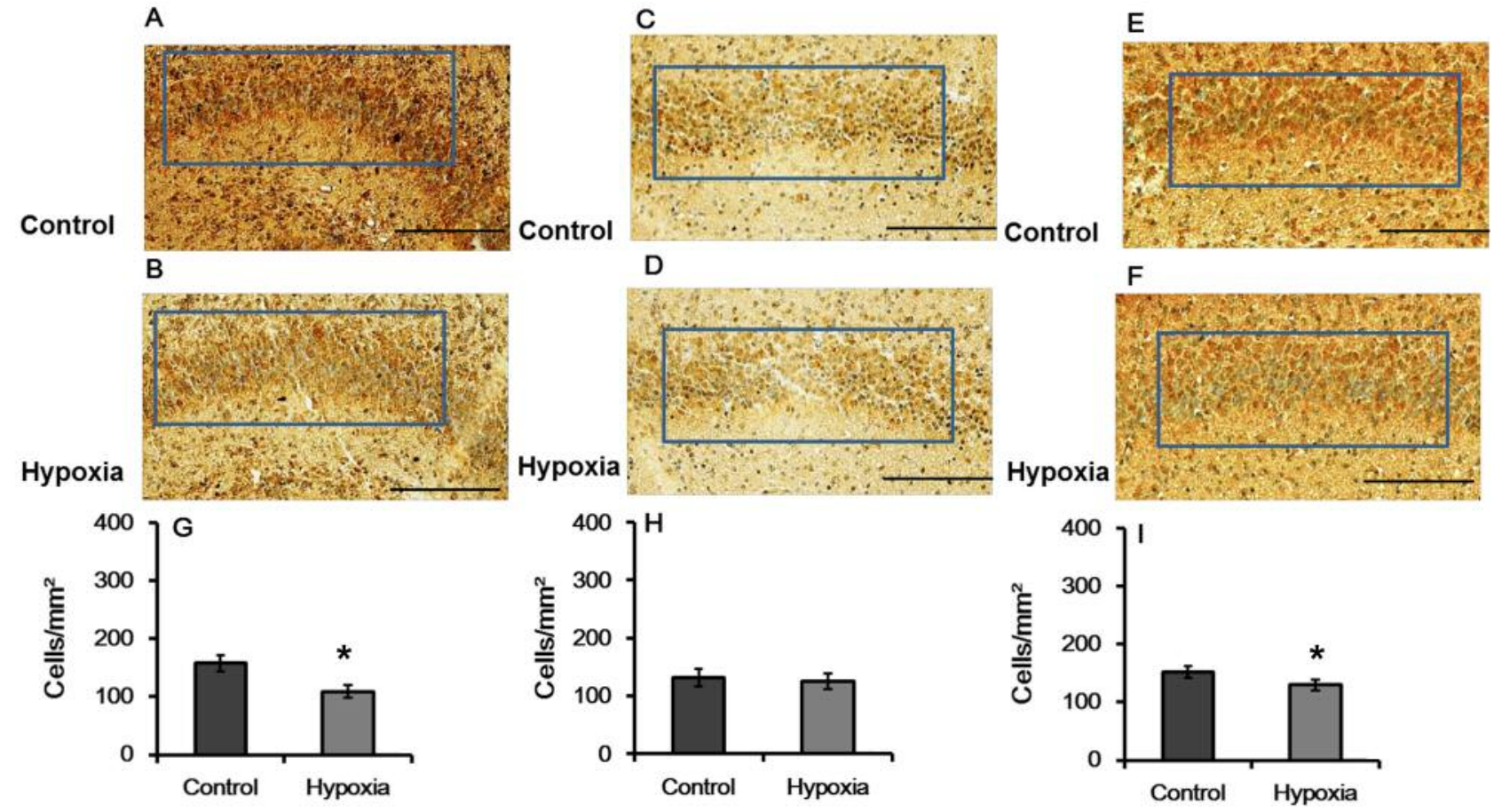

Figure 4. Representative photomicrographs of the immunoreactivity for PI3K ( $A$ and B), Akt (C and D), and pAkt $(E$ and $F)$ in the dentate gyrus (outlined by blue boxes) of rats at $21 \mathrm{dg}$. Scale bars=100 $\mu \mathrm{m}$. The densities of the PI3K-IR $(G), A k t-I R(H)$, and pAkt-IR (I) cells in the dentate gyrus of the control and hypoxic fetuses are shown, with significant decreases in the densities of the PI3K-IR and pAKT-IR cells in the hypoxic fetuses compared with the controls. The data are expressed as mean $\pm S E M . * p<0.05$.

\section{Acknowledgements}

This study was supported by research funds from Chosun University (2013).

\section{References}

1 Gross SJ, Kosmetatos N, Grimes CT and Williams ML: Newborn head size and neurological status. Predictors of growth and development of low birth weight infants. Am J Dis Child 132: 753-756, 1978.

2 Skranes J, Vangberg TR, Kulseng S, Indredavik MS, Evensen KA, Martinussen M, Dale AM, Haraldseth O and Brubakk AM: Clinical findings and white matter abnormalities seen on diffusion tensor imaging in adolescents with very low birth weight. Brain 130: 654-666, 2007.

3 Reinboth BS, Koster C, Abberger H, Prager S, Bendix I, Felderhoff-Müser U and Herz J: Endogenous hypothermic response to hypoxia reduces brain injury: Implications for modeling hypoxic-ischemic encephalopathy and therapeutic hypothermia in neonatal mice. Exp Neurol 283: 264-275, 2016.

4 Neerhof MG and Thaete LG: The fetal response to chronic placental insufficiency. Semin Perinatol 32: 201-205, 2008.

5 Liu J, Li Z and Lin Q: Intrauterine growth retardation and cerebral palsy. Zhonghua Yu Fang Yi Xue Za Zhi 35: 390-393, 2001.
6 Saigal S, Szatmari P, Rosenbaum P, Campbell D and King S: Cognitive abilities and school performance of extremely low birth weight children and matched term control children at age 8 years: a regional study. J Pediatr 118: 751-760, 1991.

7 Dringenberg HC, Richardson DP, Brien JF and Reynolds JN: Spatial learning in the guinea pig: cued versus non-cued learning, sex differences, and comparison with rats. Behav Brain Res 124: 97-100, 2001.

8 Mallard EC, Rehn A, Rees S, Tolcos M and Copolov D: Ventriculomegaly and reduced hippocampal volume following intrauterine growth-restriction: implications for the aetiology of schizophrenia. Schizophr Res 40: 11-21, 1999.

9 Daval JL and Vert P: Apoptosis and neurogenesis after transient hypoxia in the developing rat brain. Semin Perinatol 28: $257-$ 263, 2004.

10 Caviness VS Jr., Takahashi $\mathrm{T}$ and Nowakowski RS: Numbers, time and neocortical neuronogenesis: a general developmental and evolutionary model. Trends Neurosci 18 : 379-383, 1995.

11 Chung Y, So K, Kim E, Kim S and Jeon Y: Immunoreactivity of neurogenic factor in the guinea pig brain after prenatal hypoxia. Ann Anat 200: 66-72, 2015.

12 Barde YA. Neurotrophins: a family of proteins supporting the survival of neurons. Prog Clin Biol Res 390: 45-56, 1994.

13 Patapoutian A and Reichardt LF: Trk receptors: mediators of neurotrophin action. Curr Opin Neurobiol 11: 272-280, 2001. 
14 Hayashi TT and Dorko ME: A rat model for the study of intrauterine growth retardation. Am J Obstet Gynecol 158: 12031207, 1988.

15 Tolcos M, Markwick R, O'Dowd R, Martin V, Turnley A, Rees S. Intrauterine Growth Restriction: Effects on Neural Precursor Cell Proliferation and Angiogenesis in the Foetal Subventricular Zone. Dev Neurosci 37: 453-463, 2015.

16 Gilbert $\mathrm{M}$ and Leturque A: Fetal weight and its relationship to placental blood flow and placental weight in experimental intrauterine growth retardation in the rat. J Dev Physiol 4: 237246, 1982.

17 Huang EJ and Reichardt LF: Neurotrophins: roles in neuronal development and function. Annu Rev Neurosci 24: 677-736, 2001.

18 Du J, Feng L, Yang F and Lu B: Activity- and Ca(2+)-dependent modulation of surface expression of brain-derived neurotrophic factor receptors in hippocampal neurons. J Cell Biol 150: 1423$1434,2000$.

19 Mishra OP and Delivoria-Papadopoulos M: Cellular mechanisms of hypoxic injury in the developing brain. Brain Res Bull 48: 233-238, 1999.

20 Huang EJ and Reichardt LF: Trk receptors: roles in neuronal signal transduction. Annu Rev Biochem 72: 609-642, 2003.

$21 \mathrm{Jia}$ W, Feng YI, Sanders AJ, Davies EL and Jiang WG: Phosphoinositide-3-Kinase Enhancers, PIKEs: Their Biological Functions and Roles in Cancer. Anticancer Res 36: 1103-1109, 2016.

22 Crowder RJ and Freeman RS: Phosphatidylinositol 3-kinase and Akt protein kinase are necessary and sufficient for the survival of nerve growth factor-dependent sympathetic neurons. J Neurosci 18: 2933-2943, 1998.

$23 \mathrm{Kim} \mathrm{K}, \mathrm{Wu}$ HG and Jeon SR: Epidermal growth factor-induced cell death and radiosensitization in epidermal growth factor receptor-overexpressing cancer cell lines. Anticancer Res 35: 245-253, 2015.

24 Yano S, Morioka M, Fukunaga K, Kawano T, Hara T, Kai Y, Hamada J, Miyamoto E and Ushio Y: Activation of Akt/protein kinase $\mathrm{B}$ contributes to induction of ischemic tolerance in the CA1 subfield of gerbil hippocampus. J Cereb Blood Flow Metab 21: 351-360, 2001
25 Noshita N, Lewen A, Sugawara T and Chan PH: Evidence of phosphorylation of Akt and neuronal survival after transient focal cerebral ischemia in mice. J Cereb Blood Flow Metab 21: 1442-1450, 2001.

26 Kim JY, Duan X, Liu CY, Jang MH, Guo JU, Pow-anpongkul $\mathrm{N}$, Kang E, Song H and Ming GL: DISC1 regulates new neuron development in the adult brain via modulation of AKT-mTOR signaling through KIAA1212. Neuron 63: 761-773, 2009.

27 Arevalo JC and Wu SH: Neurotrophin signaling: many exciting surprises! Cell Mol Life Sci 63: 1523-1537, 2006.

28 Hirai K, Hayashi T, Chan PH, Basus VJ, James TL and Litt L: Akt phosphorylation and cell survival after hypoxia-induced cytochrome $\mathrm{c}$ release in superfused respiring neonatal rat cerebrocortical slices. Acta Neurochir Suppl 86: 227-230, 2003.

29 Ilieva H, Nagano I, Murakami T, Shiote M, Shoji M and Abe K: Sustained induction of survival p-AKT and p-ERK signals after transient hypoxia in mice spinal cord with G93A mutant human SOD1 protein. J Neurol Sci 215: 57-62, 2003.

30 Namura S, Nagata I, Kikuchi H, Andreucci M and Alessandrini A: Serine-threonine protein kinase Akt does not mediate ischemic tolerance after global ischemia in the gerbil. J Cereb Blood Flow Metab 20: 1301-1305, 2000.

31 Ouyang YB, Tan Y, Comb M, Liu CL, Martone ME, Siesjö BK and Hu BR: Survival- and death-promoting events after transient cerebral ischemia: phosphorylation of Akt, release of cytochrome $\mathrm{C}$ and Activation of caspase-like proteases. J Cereb Blood Flow Metab 19: 1126-1135, 1999. 